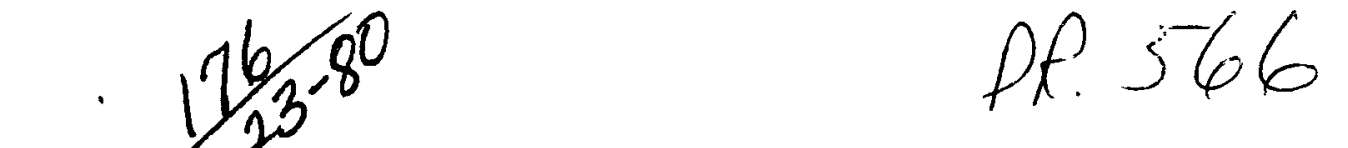

GA-A15142

UC-77

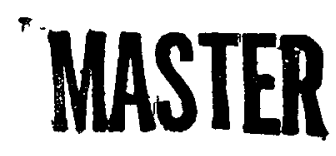

\title{
BOUNDARY VALUE PROBLEMS \\ OF THE CIRCULAR CYLINDERS \\ IN THE STRAIN-GRADIENT THEORY \\ OF LINEAR ELASTICITY
}

\author{
by \\ B. G. KAO
}

Prepared under

Contract DE-AT03-76ET35300

for the San Francisco Office

Department of Energy

DATE PUBLISHED: NOVEMBER 1979

\section{GENERAL ATOMIC COMPANY}




\title{
NOTICE
}

This report was prepared as an account of work sponsored by the United States Government. Neither the United States nor the United States Department of Energy, nor any of their employees, nor any of their contractors, subcontractors, or their employees, makes any warranty, express or implied, or assumes any legal liability or responsibility for the accuracy, completeness or usefulness of any information, apparatus, product or process disclosed, or represents that its use would not infringe privately owned rights.

\author{
Printed in the United States of America \\ Available from \\ National Technical Information Service \\ U.S. Department of Commerce \\ 5285 Port Royal Road \\ Springfield, Virginia 22161 \\ Price: Printed Copy $\$ 4.50$; Microfiche $\$ 3.00$
}




\section{DISCLAIMER}

This report was prepared as an account of work sponsored by an agency of the United States Government. Neither the United States Government nor any agency Thereof, nor any of their employees, makes any warranty, express or implied, or assumes any legal liability or responsibility for the accuracy, completeness, or usefulness of any information, apparatus, product, or process disclosed, or represents that its use would not infringe privately owned rights. Reference herein to any specific commercial product, process, or service by trade name, trademark, manufacturer, or otherwise does not necessarily constitute or imply its endorsement, recommendation, or favoring by the United States Government or any agency thereof. The views and opinions of authors expressed herein do not necessarily state or reflect those of the United States Government or any agency thereof. 


\section{DISCLAIMER}

Portions of this document may be illegible in electronic image products. Images are produced from the best available original document. 
GA-A15142

UC-77

\title{
BOUNDARY VALUE PROBLEMS \\ OF THE CIRCULAR CYLINDERS \\ IN THE STRAIN-GRADIENT THEORY \\ OF LINEAR ELASTICITY
}

\author{
by \\ B. G. KAO
}

\begin{abstract}
Prepared under
Contract DE-AT03-76ET35300

for the San Francisco Office

Department of Energy
\end{abstract}

\section{GENERAL ATOMIC PROJECT 6400 \\ DATE PUBLISHED: NOVEMBER 1979}




\section{TABLE OF CONTENTS}

ABSTRACT . . . . . . . . . . . . . . . . . . . . . $\mathrm{.}$. $\mathrm{.}$

1. INTRODUCTION . . . . . . . . . . . . . . . . . . . . . . 1

2. PRELIMINARIES . . . . . . . . . . . . . . . . . . . 4

2.1 Governing Equation . . . . . . . . . . . . . . . 4

2.2 Circular Cylindrical Coordinate System . . . . . . . . . 11

3. TWISTING OF A CIRCULAR CYLINDER . . . . . . . . . . . . . . . 15

4. CIRCULAR CYLINDER UNDER UNIFORM PRESSURE . . . . . . . . . . . 18

5. PURE-BENDING OF A SIMPLY CONNECTED CIRCULAR CYLINDER . . . . . . . 23

5.1 Method of Superposition . . . . . . . . . . . . . 23

5.2 Solution .................... . . . . 27

5.3 Stress and Bending Moment ............... 30

REFERENCES . . . . . . . . . . . . . . . . . . . 38 


\begin{abstract}
In this report, three boundary value problems in the straingradient theory of linear elasticity are solved for circular cylinders. They are the twisting of circular cylinder, uniformly pressuring of concentric circular cylinder, and pure-bending of simply connected cylinder. The comparisons of these solutions with the solutions in classical elasticity and in couple-stress theory reveal the differences in the stress fields as well as the apparent stress fuelds due to the influences of the strain-gradient. These aspects of the strain-gradient theory could be important in modeling the failure behavior of structural materials.
\end{abstract}




\section{INTRODUCTION}

In Reference 1, the material modeling of the nuclear grade graphite by the couple-stress theory of linear elasticity has been studied by comparing the experimental data and theoretical results of the purebending. It was shown that most observed mechanical responses in laboratories concerning the pure-bending of a graphite circular cylinder which are discordant in the classical elasticity are compatible with the theoretical results of the couple-stress theory. In other words, the mechanical responses of graphite beam under pure-bending can be reasonably explained in the couple-stress theory.

The couple-stress theory is restricted in the sense that it accounts only the rotation gradient of the deformation curvature which relates to the simple shear deformation. But there are experimental data of graphite that show the influences of strain-gradient when the specimen is loaded dilatationally such as the internal pressuring of a cylinder (Ref. 2). Under such loading, if the end effects are neglected, the rotation gradient vanishes and hence there is no change in stress field from the classical elastic solution according to the couple-stress theory. To investigate the influences of the strain gradient for the cylinder, it is necessary to pursue the more general strain-gradient theory of elasticity. 
In this report we adopt the strain-gradient theory of linear elasticity developed by Mindlin for the structured materials (Ref. 3). The uniqueness of the solution to the boundary value problem has been proved in a later paper of Mindlin and Eshel (Ref. 4). However, there are not many solutions to the boundary value problems available in this theory. Consequently, the physical implications and the engineering applications of the theory have not been explored comprehensively beyond its mathematical structure. The objective of this report is therefore to provide several solutions of the boundary value problems that are useful in planning the laboratory tests and in planning test results for structured materials.

The strain-gradient theory developed by Mindlin (Ref. 3) is outlined in Section 2. Some modifications, especially the boundary conditions, are incorporated according to Reference 4. For direct application to the boundary value problems discussed in this report, relevant formulae are transformed to the circular cylindrical coordinate system.

In Section 3, we deal with the twisting of a circular cylinder It is found that the displacement field in the classical elastic solution for this torsion problem is also the solution in the strain-gradient theory. Nevertheless, the torque is always greater than that predicted by the classical elasticity. The simple shear-deformation field of this solution makes the solution in strain-gradient theory identical to the solution in couple-stress theory in every respect.

The uniformly pressuring of a circular hollow cylinder is studied in Section 4. The solution reveals that because of the dilatational loading and geometric symmetry the rotation-gradient vanishes for the deformation. Consequently, the resulting stress field in symmetric. 
Comparing with classical elastic solution, which is identical to the solution in the couple-stress theory, we noticed the modifications on the stress and displacement fields.

A more general deformation - pure-bending - of a solid circular cylinder has been solved in Section 5 by the principle of superposition. The solution scheme is similar to that employed in Reference 1 and hence not all details are presented. We find further modifications in the stress field, bending moment, and displacement field over the couplestress theory.

The solutions presented in this report show the apparent modifications on the stress distribution caused by the strain-gradient. We therefore conjecture that the influence of the strain-gradient is important in the search of the failure behavior of the structured materials based on the assumption that the failure is determined by the stress field. 


\section{PRELIMINARIES}

The governing equations for the strain-gradient theory of linear elasticity of isotropic materials is outlined in this section (after Ref. 3). Some modifications are incorporated in the equations according to Mindlin and Eshe1 (Ref. 4).

The rectangular cartesian coordinate system and the index notation are adopted in the first subsection for the development of the theory. The relevant formulae are then converted to the circular cylindrical coordinate system in the second part of this section for direct applications to the boundary value problems.

\subsection{Governing Equation}

The equilibrium of a material particle in a continuous medium in the strain-gradient theory, without body forces, body couples, and body double-forces, requires that the stress tensor $\tau$ and the couple-stress tensor $\underset{\sim}{\mu}$ satisfy

$\tau_{i j, i}=0$

$\mu_{i j, i}+e_{i j k}{ }^{\tau} k i=0 \quad(i, j, k=1,2,3)$ 
where $(\cdot)_{, i}$ denotes the partial derivative with respect to $x_{i}, e_{i j k}$ is the permutation symbol, and repeated indices represent summation over 1,2 , and 3 . It is also assumed that the stress vector ${\underset{\sim}{(n)}}_{(\underset{n}{n})}$ and couplestress tensor $\mathfrak{m}_{(\mathfrak{n})}$ on a surface element with outward unit normal vector n are given by

$t_{(n) i}=n_{j} \tau_{n j}$

$m_{(n) i}=n_{j} \mu_{j i}$

Equation $2-1_{2}$ gives the condition that the stress tensor is symmetric

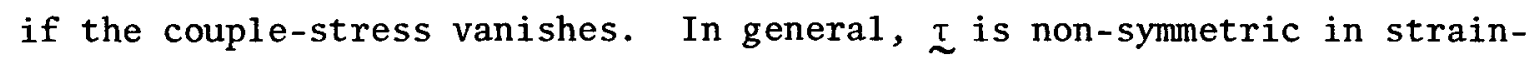
gradient theory and that its anti-symmetric part can be deduced from Eq. $2-1_{2}$ :

$$
\tau_{j k}^{A}=-\frac{1}{2} e_{i j k} \mu_{1 i, 1}
$$

Further formulation depends on the constitutive assumptions. We introduce here the constitutive assumptions for the isotropic linear elastic materials. In the strain-gradient theory, the kinematic quantities considered are the strain $\underset{\sim}{\varepsilon}$ rotation gradient $\vec{\kappa}$, and the symmetric strain-gradient $\underset{\underline{\underline{K}}}{\mathrm{~J}}$ for a given deformation $\underset{\sim}{\mathrm{u}}$. They are defined by

$$
\begin{aligned}
& \varepsilon_{i j}=\frac{1}{2}\left(u_{i, j}-u_{j, i}\right) \\
& w_{i}=\frac{1}{2} e_{i j k} u_{k, j}
\end{aligned}
$$




$$
\bar{\kappa}_{i j}=w_{j, i}
$$

$\overline{\bar{k}}_{i j k}=\frac{1}{3}\left(u_{i, j k}+u_{j, i k}+u_{k, i j}\right)$

where $\underset{\sim}{w}$ is the rotation vector.

The constitutive assumptions for the media with the influences of the strain-gradient are that the strain energy accompanying the deformation is given by

$$
\begin{array}{r}
W=\frac{\lambda}{2} \varepsilon_{i i} \varepsilon_{j j}+\mu \varepsilon_{i j} \varepsilon_{i j}+2 \bar{d}_{1} \bar{\kappa}_{i j} \bar{\kappa}_{i j}+2 \bar{d}_{2} \bar{\kappa}_{i j} \bar{\kappa}_{j i}+ \\
\frac{3}{2} \bar{a}_{1} \overline{\bar{k}}_{i i j} \overline{\bar{k}}_{k k j}+\bar{a}_{2} \overline{\bar{\kappa}}_{i j k} \overline{\bar{\kappa}}_{i j k}+\bar{f} e_{i j k} \bar{\kappa}_{i j} \overline{\bar{k}}_{k 11}
\end{array}
$$

where $\lambda$ and $\mu$ are the Lame constants, and $\bar{a}_{1}, \bar{a}_{2}, \bar{d}_{1}, \bar{d}_{2}$, and $\bar{f}$ are the material constants of the strain-gradient theory; and that the stress $\bar{\tau}$, the couple-stress $\overline{\mathcal{L}}$, and the double-stress $\overline{\mathcal{L}}$ are the partial deriva-

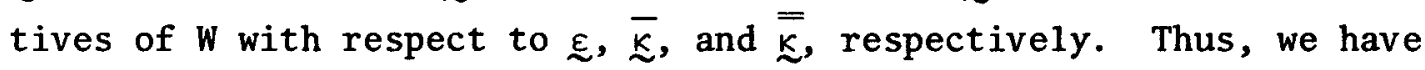

$$
\begin{aligned}
& \bar{\tau}_{i j}=\lambda \varepsilon_{k k} \delta_{i j}+2 \mu \varepsilon_{i j} \\
& \bar{\mu}_{i j}=4 \bar{d}_{1} \bar{\kappa}_{i j}+4 \bar{d}_{2} \bar{\kappa}_{j i}+\bar{f} e_{i j k} \overline{\bar{k}}_{k 11} \\
& \bar{\mu}_{i j k}=\bar{a}_{1}\left(\overline{\bar{k}}_{p p k} \delta_{i j}+\overline{\bar{k}}_{p p i} \delta_{j k}+\overline{\bar{k}}_{p p j} \delta_{i k}\right)+2 \bar{a}_{2} \bar{k}_{i j k}+ \\
& \frac{1}{3} \bar{f}_{\bar{k}_{p q}}\left(\delta_{i j} e_{p q k}+\delta_{j k} e_{p q i}+\delta_{k i} e_{p q j}\right)
\end{aligned}
$$


In these equations, we recognize that $\underset{\tau}{\tau}, \underset{\sim}{\bar{\mu}}$, and $\underset{\bar{\mu}}{\bar{\mu}}$ are defined quantities and hence, even though $\underset{\mathcal{\tau}}{\sim}$ and $\overline{\mathcal{\mu}}$ are called stress and couple-stress respectively in the rest of this report, they are not the $\tau$ and $\underset{\sim}{\sim}$ that are used in Eqs. 2-1, 2-2, and 2-3. The latter set of stress and couplestress is sometimes referred to as the actual stress and couple-stress for distinguishment. The relations between these sets of stress and couple-stress will be clarified later.

The material constants $\bar{a}_{1}, \bar{a}_{2}, \bar{d}_{1}, \bar{d}_{2}$, and $\bar{f}$ have the unit of force which can be verified from Eq. 2-6. The positive definiteness of the strain-energy $W$ for any admissible deformation yield that the material constants satisfy

$$
\begin{array}{lc}
\mu>0, & 3 \lambda+2 \mu>0, \\
\overline{\mathrm{a}}_{2}>0, & 5 \overline{\mathrm{a}}_{1}+2 \overline{\mathrm{a}}_{2}>0, \\
\overline{\mathrm{d}}_{1}>0, & \overline{\mathrm{d}}_{1}>\overline{\mathrm{d}}_{2}>-\overline{\mathrm{d}}_{1}, \\
5 \overline{\mathrm{f}}^{2}<6\left(\overline{\mathrm{d}}_{1}-\overline{\mathrm{d}}_{2}\right)\left(5 \overline{\mathrm{a}}_{1}+2 \overline{\mathrm{a}}_{2}\right)
\end{array}
$$

In terms of the Young's modulus $\mathrm{E}$ and Poisson's ratio $\nu$, since $\mathrm{E}$ and $v$ relate to the Lame constants by
$\mu=\frac{E}{2(1+\nu)}$
$\lambda=\frac{v E}{(1+\nu)(1-2 v)}$

the inequalities (Eqs. $2-7_{1}, 2-7_{2}$ ) can be replaced by 


$$
\frac{1}{2}>\nu>-1 \quad, \quad \mathrm{E}>0
$$

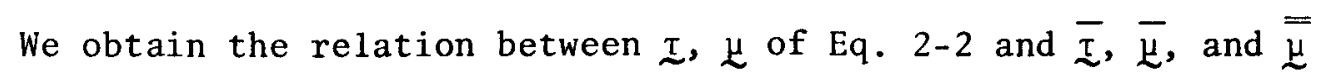
of Eq. 2-6 by the variational principle. For any admissible variation $\delta \mathfrak{d}$ of the displacement field $\underset{\sim}{\mathfrak{u}}$, the conservation of the strain-energy in a volume $v$ has the following representation:

$$
\begin{aligned}
\int\left[\bar{\tau}_{i j} \delta \varepsilon_{i j}\right. & \left.+\bar{\mu}_{i j} \delta \bar{\kappa}_{i j}+\overline{\bar{\mu}}_{i j k} \delta \overline{\bar{k}}_{i j k}\right] d v= \\
& \oint_{\partial v}\left[t_{i} \delta u_{i}+m_{i} \delta w_{i}+n_{i} \overline{\bar{\mu}}_{i j k} \delta \varepsilon_{j k}\right] d s
\end{aligned}
$$

where $\partial v$ is the surface enclosing $v$ and $\underset{\sim}{t}$ and $\underset{\sim}{m}$ are the surface force and surface couple on $\partial v$. Using the divergence theorem and the chain rule, and the relations in Eq. 2-2, the right hand side of Eq. 2-10 becomes

$$
\begin{gathered}
\int \tau_{v}\left[\tau_{i j, i} \delta u_{j}+\mu_{i j, i} \delta w_{j}+\bar{\mu}_{i j k, i} \delta \varepsilon_{j k}+\tau_{i j} \delta u_{j, i}+\right. \\
\left.\mu_{i j} \delta w_{j, i}+\bar{\mu}_{i j k} \delta \varepsilon_{j k, i}\right] d v
\end{gathered}
$$

Since $\tau_{i j} \delta u_{j, i}=\tau_{i j}^{s} \delta u_{j, i}^{s}+\tau_{i j}^{A} \delta u_{j, i}^{A}=\tau_{i j}^{s} \delta \varepsilon_{i j}+\tau_{k i} e_{i j k} \delta w_{j}$, $\mu_{i j} \delta w_{j, i}=\mu_{i j}^{D} \delta \bar{\kappa}_{i j}$, and $\overline{\bar{\mu}}_{i j k} \delta \varepsilon_{j k, i}=\bar{\mu}_{i j k} \delta \overline{\bar{k}}_{i j k}$, using Eq. $2-1$, Eq. 2-10 reduces to

$$
\int_{v}\left[\left(\bar{\tau}_{j k}-\tau_{j k}^{s}-\overline{\bar{\mu}}_{i j k, i}\right) \delta \varepsilon_{j k}+\left(\bar{\mu}_{j k}-\mu_{j k}^{D}\right) \delta \bar{k}_{j k}\right] d v=0
$$


In the above expressions, the superscripts $s, A$, and $D$ denote the symmetric part, the anti-symmetric part, and the deviator of the tensors, respectively. The standard argument in the calculus of variation then yields:

$$
\begin{aligned}
& \tau_{j k}^{s}=\bar{\tau}_{j k}-\overline{\bar{\mu}}_{i j k, i} \\
& \mu_{j k}^{D}=\bar{\mu}_{j k}
\end{aligned}
$$

Equation $2-12_{2}$ further yields the decomposition of $\underset{\sim}{\mu}:$

$$
\mu_{i j}=\bar{\mu}_{i j}+\frac{1}{3} \delta_{i j} \mu_{k k}
$$

The equations of equilibrium (Eq. 2-1) can then be expressed in terms of $\bar{\tau}, \underset{\sim}{\sim}$, and $\underset{\sim}{\bar{\mu}}$ by using Eqs. 2-3, 2-12, and 2-13. Observing that $e_{i j k}{ }_{11, k j}=0$, one obtains

$$
\bar{\tau}_{i j, i}-\overline{\bar{\mu}}_{1 i j, 1 i}-\frac{1}{2} e_{1 i j} \bar{\mu}_{k 1, k i}=0
$$

In terms of the deformation $\underset{\sim}{u}$, we substitute Eqs. 2-4 and 2-6 into Eq. 2-14, which becomes, in vector notation,

$$
(\lambda+2 \mu)\left(1-\ell_{1}^{2} \nabla^{2}\right) \nabla \nabla \cdot \underline{\sim}-\mu\left(1-\ell_{2}^{2} \nabla^{2}\right) \nabla \times \nabla \times \underset{\sim}{u}=0
$$


where $\ell_{1}$ and $\ell_{2}$ are the characteristic lengths of the strain-gradient theory defined by

$\ell_{1}^{2}=\frac{3 \bar{a}_{1}+2 \bar{a}_{2}}{\lambda+2 \mu}, \quad \ell_{2}^{2}=\frac{3 \bar{d}_{1}+\bar{a}_{1}+2 \bar{a}_{2}-\bar{f}}{3 \mu}$

It follows from Eq. 2-7 that $\ell_{1}^{2}$ and $\ell Z$ are both positive.

Since $\mu_{k k}$ appears neither in the equation of equilibrium (Eq. 2-14) nor in the internal energy equation (Eq. 2-5), it is a function of position solely. Therefore, $\mu_{k k}$ is important only in the satisfaction of the boundary conditions. For this reason, it is convenient to adjust the boundary conditions so the convention

$\mu_{\mathbf{k k}}=0$

can be employed in the formulation of the strain-gradient theory. Consequently, the stress tensor $\tau$ has entries of the form:

$\tau_{j k}=\bar{\tau}_{j k}-\overline{\bar{\mu}}_{i j k, i}-\frac{1}{2} e_{1 j k} \bar{\mu}_{i 1, i}$

The surface traction boundary conditions are obtained by using the principle of virtual work for the whole body. For the given surface force $\underset{\sim}{P}$, surface couple $\bar{Q}$ that tangent to the surface (the normal component is left out here because the adoption of Eq. 2-17), surface double-force $\bar{R}$ that normal to the surface and the force $\underset{\sim}{\mathbb{E}}$ that applied on the edge of the bounding surface, the boundary conditions are, in the vector notation: 


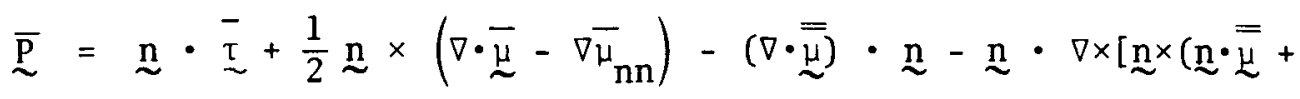

$$
\begin{aligned}
& \left.\underset{\sim}{n} \cdot \overline{\bar{\mu}} \cdot \mathrm{nn}^{\prime}\right]
\end{aligned}
$$

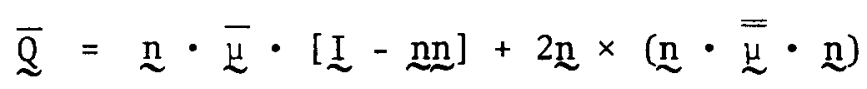

$$
\begin{aligned}
& \overline{\mathrm{R}}=\underline{\mathrm{n}} \cdot(\underline{\mathrm{n}} \cdot \underset{\sim}{\underline{\mu}} \cdot \mathfrak{n}) \\
& \overline{\mathrm{E}}=\llbracket\left[\frac{1}{2} \mu_{\mathrm{nn}} \underline{\mathbf{s}}+(\underline{\underline{s}} \times \underline{\mathrm{n}}) \cdot(\underset{\sim}{\mathrm{n}} \cdot \overline{\bar{\mu}}+\underline{\underline{n}} \cdot \overline{\bar{\mu}} \cdot \underline{\mathrm{nn}}) \rrbracket\right.
\end{aligned}
$$

where $\underset{\sim}{n}$ is the unit normal vector on the surface, $\bar{\mu}_{n n}=\underline{n} \cdot \bar{L} \cdot \underset{\sim}{n}$ is a scalar function, $\underset{\sim}{\sim}$ is the identity matrix, $\underset{\sim}{n}$ is the dyadic product of $\mathfrak{\sim}$ with itself, $s$ is the unit tangent vector along the edge and that $[\mathbb{]}]$ denotes the difference across the edge for the quantity enclosed.

It is clear from Eqs. 2-6, 2-14, and 2-19 that the couple-stress theory (Ref. 5) is a special case of the strain-gradient theory with the constants $\bar{a}_{1}, \bar{a}_{2}$, and $\bar{f}$ vanish.

\subsection{Circular Cylindrical Coordinate System}

For the boundary value problems of circular cylinders discussed in this article it is convenient to employ the circular cylindrical coordinate system $(r, \theta, z)$ so that the $z$-axis coincides with the central axis of the cylinder. Let $\left[\mathfrak{\sim}^{<r>}, \mathfrak{\sim}^{<\theta>}, \mathfrak{e}^{<z>}\right]$ be the orthonormal basis at an arbitrary point $(r, \theta, z)$, then the displacement vector $\underset{\sim}{u}$ has the representation 
$\underset{\sim}{u}=u_{r} \stackrel{e}{ }^{<r>}+u_{\theta} \stackrel{e}{e}^{\left\langle\theta>+u_{z}\right.} \stackrel{e}{<}<z>$

where $u_{r}, u_{\theta}$, and $u_{z}$ are functions of position.

The formulae in the previous subsection can be transformed to the cylindrical coordinates by recognizing that the partial derivatives in the cartesian rectangular coordinate system is the covariant derivatives in the curvilinear coordinate system. For instance, the symmetric strain-gradient $\overline{\bar{k}}$ has the following entries:

$$
\begin{aligned}
& \overline{\bar{k}}_{r r r}=u_{r, r r} \\
& \overline{\bar{k}}_{\theta r r}=\frac{1}{3}\left(u_{\theta, r r}-\frac{2}{r} u_{\theta, r}+\frac{2}{r^{2}} u_{\theta}+\frac{2}{r} u_{r, r \theta}-\frac{2}{r^{2}} u_{r, \theta}\right) \\
& \overline{\bar{k}}_{z r r}=\frac{1}{3}\left(u_{z, r r}+2 u_{r, r z}\right) \\
& \overline{\bar{k}}_{r \theta \theta}=\frac{1}{3}\left(\frac{3}{r^{\prime}} u_{r, r}+\frac{1}{r^{2}} u_{r, \theta \theta}-\frac{3}{r^{2}} u_{r}+\frac{2}{r} u_{\theta, \theta r}-\frac{4}{r^{2}} u_{\theta, \theta}\right) \\
& \overline{\bar{k}}_{\theta \theta \theta}=\frac{2}{r^{2}} u_{r, \theta}+\frac{1}{r^{2}} u_{\theta, \theta \theta}-\frac{1}{r^{2}} u_{\theta}+\frac{1}{r} u_{\theta, r} \\
& \overline{\bar{k}}_{z \theta \theta}=\frac{1}{3}\left(\frac{1}{r^{2}} u_{z, \theta \theta}+\frac{1}{r} u_{z, r}+\frac{2}{r} u_{r, z}+\frac{2}{r} u_{\theta, \theta z}\right) \\
& \overline{\bar{k}}_{r z z}=\frac{1}{3}\left(u_{r, z z}+2 u_{z, z r}\right)
\end{aligned}
$$




$$
\begin{aligned}
& \overline{\bar{\kappa}}_{\theta z z}=\frac{1}{3}\left(u_{\theta, z z}+\frac{2}{r} u_{z, z r}\right) \\
& \overline{\bar{k}}_{z z z}=u_{z, z z} \\
& \overline{\bar{k}}_{r \theta z}=\frac{1}{3}\left(\frac{1}{r} u_{r, \theta z}+u_{\theta, r z}-\frac{1}{r} u_{\theta, z}+\frac{1}{r} u_{z, \theta r}-\frac{1}{r^{2}} u_{z, \theta}\right)
\end{aligned}
$$

The strain $\underset{\sim}{\varepsilon}$ and rotation gradient $\underset{\sim}{\overline{\mathcal{K}}}$ have been given in Reference 1 and the rest of the equations in Section 2.1 can be used directly.

For direct applications, the boundary conditions (Eq. 2-19) are expanded in the cylindrical coordinates as follows:

$$
\begin{aligned}
& \overline{\mathrm{P}}_{\mathrm{r}}=\bar{\tau}_{\mathrm{rr}}-\frac{\partial \overline{\bar{\mu}}_{\mathrm{rrr}}}{\partial \mathrm{r}}-\frac{3}{\mathrm{r}} \frac{\partial \overline{\bar{\mu}}_{\mathrm{rr} \theta}}{\partial \theta}-3 \frac{\partial \overline{\bar{\mu}}_{\mathrm{rrz}}}{\partial z}-\frac{1}{\mathrm{r}} \overline{\bar{\mu}}_{\mathrm{rrr}}+\frac{3}{\mathrm{r}} \overline{\bar{\mu}}_{\mathrm{r} \theta \theta} \\
& \overline{\mathrm{P}}_{\theta}=\bar{\tau}_{\mathrm{r} \theta}+\frac{1}{2} \frac{\partial \bar{\mu}_{\mathrm{rr}}}{\partial z}-\frac{1}{2}\left(\frac{\bar{\mu}_{\mathrm{rz}}}{\partial \mathrm{r}}+\frac{1}{\mathrm{r}} \frac{\partial \bar{\mu}_{\theta z}}{\partial \theta}+\frac{\partial \bar{\mu}_{\mathrm{zz}}}{\partial z}+\frac{1}{\mathrm{r}} \bar{\mu}_{\mathrm{rz}}\right)- \\
& \frac{\partial \overline{\bar{\mu}}_{r r \theta}}{\partial r}-\frac{2}{r} \frac{\partial \overline{\bar{\mu}}_{r \theta \theta}}{\partial \theta}-2 \frac{\partial \overline{\bar{\mu}}_{r \theta z}}{\partial z}-4 \overline{\bar{\mu}}_{r r \theta}+\frac{1}{r} \overline{\bar{\mu}}_{\theta \theta \theta} \\
& \overline{\mathrm{P}}_{z}=\bar{\tau}_{\mathrm{rz}}-\frac{1}{2 \mathrm{r}} \frac{\partial \overline{\bar{\mu}}_{\mathrm{rr}}}{\partial \theta}+\frac{1}{2}\left(\frac{\partial \bar{\mu}_{\mathrm{r} \theta}}{\partial \mathrm{r}}+\frac{1}{\mathrm{r}} \frac{\partial \bar{\mu}_{\theta \theta}}{\partial \theta}+\frac{\partial \bar{\mu}_{z \theta}}{\partial z}+\frac{1}{\mathrm{r}} \bar{\mu}_{\mathrm{r} \theta}+\frac{1}{\mathrm{r}} \bar{\mu}_{\theta \mathrm{r}}\right)- \\
& \frac{\partial \overline{\bar{\mu}}_{\mathrm{rrz}}}{\partial \mathrm{r}}-\frac{2}{\mathrm{r}} \frac{\partial \overline{\bar{\mu}}_{\mathrm{r} \theta z}}{\partial \theta}-2 \frac{\partial \overrightarrow{\bar{\mu}}_{\mathrm{rzz}}}{\partial z}-\frac{1}{\mathrm{r}} \stackrel{\bar{\mu}}{\mathrm{rrz}}^{\mathrm{m}}+\frac{1}{\mathrm{r}} \overline{\bar{\mu}}_{\theta \theta z}
\end{aligned}
$$




$$
\begin{aligned}
& \bar{Q}_{\theta}=\bar{\mu}_{r \theta}-2 \overline{\bar{\mu}}_{r r z} \\
& \bar{Q}_{z}=\bar{\mu}_{r z}+2 \bar{\mu}_{r r \theta} \\
& \bar{R}=\overline{\bar{\mu}}_{r r r} \\
& \underset{\sim}{\bar{E}}=0
\end{aligned}
$$

In Eq. 2-227, we have assumed that the cylinder is long enough that the end effects can be neglected and the cylinder can be considered as infinite in all practical purposes.

In sections that follow, we discuss several boundary value problems of the circular cylinders by utilizing the governing equations presented in this section. For each problem, the displacement field, the stress field, and other relevant physical quantities are calculated and presented. 


\section{TWISTING OF A CIRCULAR CYLINDER}

The first boundary value problem of our investigation is a long circular cylinder twisted by end torques. The solution presented here also serves as an example in clarifying the role of $\mu_{k k}$ in the satisfaction of the boundary conditions.

Let us consider a uniform circular cylinder of radius a is twisted by equal but opposite end torques while the cylinder surface is free from surface tractions. Thus the left hand sides of Eq. 2-22 vanish for the boundary conditions. Such a deformation can be represented by the displacement vector (Eq. 2-20) with

$u_{r}=u_{z}=0, \quad u_{\theta}=\phi r z$

where $\phi$ is a constant corresponding to the twisting angle per unit length of the cylinder. We remark here that Eq. 3-1 is taken from the classical solution of elasticity for the twisting of a circular cylinder (Ref.6). The satisfaction of Eq. 2-15 can be verified easily. The condition for Eq. 3-1 being a solution to the boundary problem is thus the satisfaction of the traction boundary conditions on the cylinder surface $r=a$.

First, we compute the strain and strain-gradients for the deformation field (Eq. 3-1) and find the following non-zero entries:

$$
\varepsilon_{\theta z}=\frac{1}{2} \phi r
$$


$\bar{\kappa}_{r r}=\bar{\kappa}_{\theta \theta}=-\frac{1}{2} \phi, \quad \bar{\kappa}_{z z}=\phi$

Thus, the stress and couple-stress can be calculated from Eq. 2-6:

$\bar{\tau}_{\theta z}=\mu \phi r$

$\bar{\mu}_{r r}=\bar{\mu}_{\theta \theta}=-2\left(\bar{d}_{1}+\bar{d}_{2}\right) \phi \quad, \quad \bar{\mu}_{z z}=4\left(\bar{d}_{1}+\bar{d}_{2}\right) \phi$

The remaining entries of $\bar{\tau}, \bar{\mu}$, and $\underset{\bar{\mu}}{\sim}$ are all zeros. The vanishing of $\underset{\sim}{\bar{\mu}}$ reflects the fact that $\underset{\sim}{\mathrm{u}}$ described by Eq. 3-1 is a simple shear deformation.

The boundary conditions (Eq. 2-22) are all satisfied on the surface $r=a$ which can be verified by the direct substitution of Eq. 3-3. However, we note from Eq. 3-3 that $\bar{\mu}_{\mathrm{rr}} \neq 0$. Thus, the solution of Eq. 3-1 with the convention of Eq. 2-17 corresponds to the end torques and a surface couple normal to the cylinder surface. In order to force the satisfaction of the zero surface couple, we remove the convention of Eq. 2-17 and require the zero surface traction condition being satisfied, i.e.:

$\mu_{r r}=\mu_{k k}-2\left(\bar{d}_{1}+\bar{d}_{2}\right) \phi=0$

Consequently, we obtain from Eq. 2-18 that

$\mu_{\theta \theta}=0, \quad \mu_{z z}=6\left(\bar{d}_{1}+\bar{d}_{2}\right) \phi$ 
Now that we have confirmed Eq. 3-1 as being the solution in the straingradient theory and that we have obtained the stress and couple-stress fields, it will be interesting to demonstrate the difference between the strain-gradient theory and the classical elasticity through the total torque in the cylinder. In the strain-gradient theory, the torque $\mathrm{T}$ in the circular cylinder is defined as

$$
\begin{aligned}
\underset{\sim}{\mathrm{T}} & \equiv \int_{\mathrm{A}}[\underline{\mathrm{r}} \times(\underline{\mathrm{n}} \cdot \tau)+\underline{\sim} \cdot \underline{\sim}] \mathrm{ds} \\
& =\mathrm{e}^{<z>} \int_{0}^{\mathrm{a}} \int_{0}^{2 \pi}\left(\tau_{\theta z} r+\mu_{z z}\right) \operatorname{rdrd} \theta
\end{aligned}
$$

where in Eq. 3-6 2 A has been chosen as the orthogonal plane to the cylinder axis and hence $\mathfrak{\sim}^{<z>}$ is the unit normal vector to the cross section. We have also used the stress and couple-stress fields as given by Eqs. 3-3, 2-18, and 3-5 while all other entries are zeros. Substituting Eqs.3-3 and $3-5_{2}$ into Eq. 3-6, we obtain:

$\stackrel{T}{\sim}=2 \pi \phi\left[\frac{\mu}{4} a^{4}+3\left(\bar{d}_{1}+\bar{d}_{2}\right) a^{2}\right] \stackrel{e}{<}^{<>}$

In this torque, the first term is the classical elastic twisting moment while the second term, which is proportional to the cylinder area, is the contribution due to the couple-stresses. By the inequalities (Eq s. 2-76 and 2-77), the contribution of the couple-stresses is always strengthening the cylinder in the deformation. We also remark that since the symmetric strain-gradient vanishes, the solution here is identical to the solution in the couple-stress theory. 


\section{CIRCULAR CYLINDER UNDER UNIFORM PRESSURE}

Consider a rectilinear circular tube of internal radius $r_{i}$ and external radius $r_{0}$ loaded with the uniform internal pressure $p_{i}$, external pressure $\mathrm{p}_{0}$, and with an axial load $\mathrm{p}_{z}$. If the tube is fairly long so the corresponding boundary value problem can be considered as an infinite circular cylinder in analysis, then the symmetries of the geometry and the loading infer that the displacement field $\underset{\sim}{u}$ of Eq. 2-20 for the cylinder can be represented by:
$u_{r}=u_{r}(r)$,
$u_{\theta}=0$,
$u_{z}=\varepsilon z$

where $u_{r}$ is a function of $r$ solely and $\varepsilon$ is a constant that represents the axial strain of the cylinder. The displacement field of Eqs.4-1 indicates a generalized plane-strain deformation field.

The differential equations for $u_{r}$ are obtained by substituting Eq. 2-20 with Eq. 4-1 into Eq. 2-15. The solution for the resulting ordinary differential equation is:

$u_{r}=A \ell_{1} I_{1}\left(\frac{r}{\ell_{1}}\right)+B \ell_{1} K_{1}\left(\frac{r}{\ell_{1}}\right)+C \ell_{1}^{2} \frac{1}{r}+D r$

where A, B, C, and D are the normalized constants of integration so that they are absolute numbers and $I_{1}(\cdot)$ and $K_{1}(\cdot)$ are the modified Bessel 
functions of the first and the second kinds of the order 1, respectively. We note that the solution of Eq. 4-2 is independent of $\ell_{2}$ which reflects the dilatational deformation of the displacement of Eq. 4-1.

For the deformation of Eq. 4-1 with $u_{r}$ given by Eq. 4-2, we can ca1culate the strain and the curvatures and hence the stress $\bar{\tau}$, the couplestress $\underset{\sim}{\tilde{\mu}}$, and the double-stress $\underset{\sim}{\underline{\sim}}$. The actual stress tensor $\underset{\sim}{\sim}$ will then be obtained by using Eq. 2-18. The non-zero entries of $\underset{\sim}{\sim}$ and $\underset{\bar{\mu}}{\sim}$ are listed in the following with the integration constants $A, B, C$, and $D$ and the axial strain $\varepsilon$ to be determined later:

$\bar{\mu}_{\theta z}=-\bar{\mu}_{z \theta}=\bar{f} \frac{1}{\ell_{1}}\left[A I_{1}\left(\frac{r}{\ell_{1}}\right)+B K_{1}\left(\frac{r}{\ell_{1}}\right)\right]$

$$
\begin{aligned}
\overline{\bar{\mu}}_{\mathrm{rrr}}=\frac{\mathrm{A}}{\ell_{1}}\left\{\left[(\lambda+2 \mu) \ell_{1}^{2}+4 \overline{\mathrm{a}}_{2} \frac{\ell_{1}^{2}}{\mathrm{r}^{2}}\right] \mathrm{I}_{1}\left(\frac{\mathrm{r}}{\ell_{1}}\right)-2 \overline{\mathrm{a}}_{2} \frac{\ell_{1}}{\mathrm{r}} \mathrm{I}_{0}\left(\frac{\mathrm{r}}{\ell_{1}}\right)\right\}+ \\
\frac{\mathrm{B}}{\ell_{1}}\left\{\left[(\lambda+2 \mu) \ell_{1}^{2}+4 \overline{\mathrm{a}}_{2} \frac{\ell^{2}}{\mathrm{r}^{2}}\right] \mathrm{K}_{1}\left(\frac{\mathrm{r}}{\ell_{1}}\right)+2 \overline{\mathrm{a}}_{2} \frac{\ell_{1}}{\mathrm{r}} \mathrm{K}_{0}\left(\frac{\mathrm{r}}{\ell_{1}}\right)\right\}+ \\
\text { 4C } \overline{\mathrm{a}}_{2} \frac{\ell_{1}^{2}}{\mathrm{r}^{3}}
\end{aligned}
$$

$$
\begin{aligned}
\overline{\bar{\mu}}_{\mathrm{r} \theta \theta}=\frac{\mathrm{A}}{\ell_{1}} & \left\{2 \overline{\mathrm{a}}_{2} \frac{\ell_{1}}{\mathrm{r}} \mathrm{I}_{0}\left(\frac{\mathrm{r}}{l_{1}}\right)+\left(\overline{\mathrm{a}}_{1}-4 \overline{\mathrm{a}}_{2} \frac{\ell^{2}}{\mathrm{r}^{2}}\right) \mathrm{I}_{1}\left(\frac{\mathrm{r}}{\ell_{1}}\right)\right\}- \\
& \frac{\mathrm{B}}{\ell_{1}}\left\{2 \overline{\mathrm{a}}_{2} \frac{\ell_{1}}{\mathrm{r}} \mathrm{K}_{0}\left(\frac{\mathrm{r}}{\ell_{1}}\right)-\left(\overline{\mathrm{a}}_{1}-4 \overline{\mathrm{a}}_{2} \frac{\ell_{1}^{2}}{\mathrm{r}^{2}}\right) \mathrm{K}_{1}\left(\frac{\mathrm{r}}{\ell_{1}}\right)\right\}- \\
4 \mathrm{C} & \overline{\mathrm{a}}_{2} \frac{\ell_{1}^{2}}{\mathrm{r}^{3}}
\end{aligned}
$$




$$
\overline{\bar{\mu}}_{\mathrm{rzz}}=\overline{\mathrm{a}}_{1} \frac{1}{\ell_{1}}\left[\mathrm{~A} \mathrm{I}_{1}\left(\frac{\mathrm{r}}{\ell_{1}}\right)+\mathrm{B} \mathrm{K}_{1}\left(\frac{\mathrm{r}}{\ell_{1}}\right)\right]
$$

The actual stress $\underset{\sim}{\sim}$ after some simplifications by using Eq. 2-16, will be able to be expressed as

$$
\begin{aligned}
& \tau_{\mathrm{rr}}=2\left(\frac{\overline{\mathrm{a}}_{1}+\overline{\mathrm{a}}_{2}}{\ell_{\mathrm{I}}^{2}}-\mu\right)\left[\mathrm{A} \frac{\ell_{1}}{\mathrm{r}} \mathrm{I}_{1}\left(\frac{\mathrm{r}}{\ell_{1}}\right)+B \frac{\ell_{1}}{\mathrm{r}} \mathrm{K}_{1}\left(\frac{\mathrm{r}}{\ell_{1}}\right)\right]+ \\
& 2(\lambda+\mu) D-2 \mu C \frac{\ell^{2}}{\mathrm{r}^{2}}+\lambda \varepsilon \\
& \tau_{\theta \theta}=A\left[\left(\lambda-\frac{\overline{\mathbf{a}}_{1}}{\ell_{1}^{2}}\right) I_{0}\left(\frac{\mathbf{r}}{\ell_{1}}\right)+2\left(\mu-\frac{\overline{\mathbf{a}}_{1}+\overline{\mathbf{a}}_{2}}{\ell_{1}^{2}}\right) \frac{\ell_{1}}{\mathbf{r}} I_{1}\left(\frac{\mathbf{r}}{l_{1}}\right)\right]- \\
& \text { B }\left[\left(\lambda-\frac{\bar{a}_{1}}{\ell_{1}}\right) K_{0}\left(\frac{r}{\ell_{1}}\right)-2\left(\mu-\frac{\bar{a}_{1}+\bar{a}_{2}}{\ell_{1}^{2}}\right) \frac{\ell_{1}}{r} K_{1}\left(\frac{r}{\ell_{1}}\right)\right]+ \\
& 2(\lambda+\mu) D+2 \mu C \frac{l^{2}}{\mathrm{r}^{2}}+\lambda \varepsilon \\
& \tau_{z z}=\left(\lambda-\frac{\bar{a}_{1}}{\ell_{1}}\right)\left[A I_{0}\left(\frac{r}{\ell_{1}}\right)-B K_{0}\left(\frac{r}{\ell_{1}}\right)\right]+2 \lambda D+(\lambda+2 \mu) \varepsilon \\
& { }^{\tau} r \theta=\tau_{\theta r}={ }^{\tau_{r z}}=\tau_{z r}=\tau_{\theta z}=\tau_{z \theta}=0
\end{aligned}
$$

We observe that there is no influence of the couple-stresses on the actual stress (Eq. 4-4) and hence $\tau$ is symmetric. We also note that the couple-stress given by Eqs. $4-3_{1}$ and $4-3_{2}$ correspond to zero normal surface couple and hence Eq. $2-17$ is satisfied and $\underline{\sim}=\underline{\sim}$. 
Next, we determine the integration constants. First, for a given axial strain $\varepsilon$, the integration constants $A, B, C$, and $D$ can be uniquely determined by using the boundary conditions (Eq. 2-22). The surface force $\overline{\mathrm{P}}$ gives two relations on the cylinder surfaces:

$$
\begin{gathered}
\overline{\mathrm{P}}_{\mathrm{r}}=\mathrm{A}\left[2 \frac{\overline{\mathrm{a}}_{2}}{\mathrm{r}^{2}} \mathrm{I}_{0}\left(\frac{\mathrm{r}}{\ell_{1}}\right)+\left(\lambda-4 \frac{\overline{\mathrm{a}}_{2}}{\mathrm{r}^{2}}\right) \frac{\ell_{1}}{\mathrm{r}} \mathrm{I}_{1}\left(\frac{\mathrm{r}}{\ell_{1}}\right)\right]-\mathrm{B}\left[2 \frac{\overline{\mathrm{a}}_{2}}{\mathrm{r}^{2}} \mathrm{~K}_{0}\left(\frac{\mathrm{r}}{\ell_{1}}\right)-\right. \\
\left.\left(\lambda-4 \frac{\overline{\mathrm{a}}_{2}}{\mathrm{r}^{2}}\right) \frac{\ell_{1}}{\mathrm{r}} \mathrm{K}_{1}\left(\frac{\mathrm{r}}{\ell_{1}}\right)\right]-\mathrm{C}\left(2 \mu+4 \frac{\overline{\mathrm{a}}_{2}}{\mathrm{r}^{2}}\right) \frac{\ell_{1}^{2}}{\mathrm{r}^{2}}+2 \mathrm{D}(\lambda+2 \mu)+\varepsilon \mu \\
\text { at } \mathrm{r}=\mathrm{r}_{\mathrm{i}} \quad \text { and } \quad \mathrm{r}=\mathrm{r}_{0}
\end{gathered}
$$

where $\overline{\mathrm{P}}_{r}=-\mathrm{p}_{i}$ at $\mathrm{r}=\mathrm{r}_{i}$ and $\overline{\mathrm{P}}_{\mathrm{r}}=-\mathrm{p}_{0}$ at $\mathrm{r}=\mathrm{r}_{0}$, respectively. The remaining non-trivial boundary conditions are given by Eq. $2-226$, i.e., by using Eq. $4-3_{2}$ :

$$
\begin{gathered}
0=A\left[\left(\lambda+2 \mu+4 \frac{\bar{a}_{2}}{\mathrm{r}^{2}}\right) I_{1}\left(\frac{\mathrm{r}}{\ell_{1}}\right)-2 \frac{\overline{a_{2}}}{\mathrm{r}_{1}} \mathrm{I}_{0}\left(\frac{\mathrm{r}}{\ell_{1}}\right)\right]+B[(\lambda+2 \mu+ \\
\left.\left.4 \frac{\overline{\mathrm{a}}_{2}}{\mathrm{r}^{2}}\right) \mathrm{~K}_{1}\left(\frac{\mathrm{r}}{\ell_{1}}\right)+2 \frac{\overline{\mathrm{a}}_{2}}{\mathrm{r}_{1}} \mathrm{~K}_{0}\left(\frac{\mathrm{r}}{\ell_{1}}\right)\right]+4 \mathrm{C} \overline{\mathrm{a}}_{2} \frac{\ell_{1}}{\mathrm{r}^{3}} \\
\text { at } \mathrm{r}=\mathrm{r}_{\mathrm{i}} \text { and } \mathrm{r}=\mathrm{r}_{\mathrm{o}}
\end{gathered}
$$

Equations 4-5 and 4-6 each have two linearly independent algebraic equations for the determinations of the constants A, B, C, and D. Therefore, for a known $E, A, B, C$, and $D$ are uniquely determined from Eqs. 4-5 and 4-6. 
If $\varepsilon$ is not known in advance, such as an axial load $P_{z}$ is given instead, an additional constraint is then needed in uniquely characterizing the five unknowns. We adopt the condition that the axial load $\mathrm{P}_{\mathrm{Z}}$ is the summation of the stress in the $z$-direction, i.e.,

$P_{z}=\int_{0}^{2 \pi} \int_{r_{i}}^{r_{0}} \tau_{z z} r d \theta d r$

Plugging Eq. 4-4 3 into Eq. 4-7 and executing the integration, we then obtain:

$$
\begin{array}{r}
P_{z}=2 \pi\left(\lambda \ell_{1}^{2}-\bar{a}_{1}\right)\left\{A\left[\frac{r_{0}}{\ell_{1}} I_{1}\left(\frac{r_{0}}{\ell_{1}}\right)-\frac{r_{i}}{\ell_{1}} I_{1}\left(\frac{r_{i}}{\ell_{1}}\right)\right]+B\left[\frac{r_{0}}{\ell_{1}} K_{1}\left(\frac{r_{0}}{\ell_{1}}\right)-\right.\right. \\
\left.\left.\frac{r_{i}}{\ell_{1}} K_{1}\left(\frac{r_{i}}{\ell_{1}}\right)\right]\right\}+\pi\left(r_{0}^{2}-r_{i}^{2}\right)[2 \lambda E+(\lambda+2 \mu) \varepsilon]
\end{array}
$$

The five constants can thus be determined uniquely from Eqs. 4-5, 4-6, and 4-8.

It is easy to observe that the solution of the same problem in the classical elasticity (Ref. 6) is a special case of Eq. 4-2 with $\overline{\mathrm{a}}_{1}=\overline{\mathrm{a}}_{2}=\overline{\mathrm{d}}_{1}=\overline{\mathrm{d}}_{2}=\overline{\mathrm{f}}=0$. Furthermore, the solution of the couple-stress theory is identical to the classical elastic solution because $\bar{d}_{1}$ and $\bar{d}_{2}$ do not appear in the solution (Eq. 4-2). 
5. PURE-BENDING OF A SIMPLY CONNECTED CIRCULAR CYLINDER

\subsection{Method of Superposition}

In this section, we follow the procedure of superposition employed in Reference 1 to solve for the pure-bending of a circular cylinder in the strain-gradient theory of linear elasticity.

Let a simply connected circular cylinder of radius a be loaded on the ends by a pair of equal but opposite bending moments. Let a rectangular cartesian coordinate system be chosen in such a way that the $z$-axis coincides with the central axis of the cylinder and that the $y$-axis is opposite to the moment vector on the normal cross section whose normal vector is in the positive z-direction. In terms of the circular cylindrical coordinate system with $\theta=0$ direction agrees with the $x$-axis, disregarding the possible rigid body motions, we search a displacement field of the form Eq. 2-20 with

$$
\begin{aligned}
& u_{r}=-\frac{1}{2}\left(v r^{2}+z^{2}\right) \frac{\cos \theta}{\rho}+u_{r}^{(1)}(r, \theta) \\
& u_{\theta}=-\frac{1}{2}\left(v r^{2}-z^{2}\right) \frac{\sin \theta}{\rho}+u_{\theta}^{(1)}(r, \theta) \\
& u_{z}=\operatorname{zr} \frac{\cos \theta}{\rho}
\end{aligned}
$$


where $\rho$ is the radius of bending curvature for the cylinder and $u_{r}^{(1)}(\cdot, \cdot)$ and $u_{\theta}^{(1)}(\cdot, \cdot)$ are functions of $r$ and $\theta$ solely.

In Eq. 5-1, $-\frac{1}{2}\left(\nu \mathrm{r}^{2}+\mathrm{z}^{2}\right) \frac{\cos \theta}{\rho}$ etc. are the classical elastic solution of the pure-bending (Ref. 6) and $\underset{\sim}{u}(1)=u_{r}^{(1)} \underset{\sim}{e}\left\langle r>+u_{\theta}^{(1)} \underset{\sim}{e}<\theta>\right.$ is a generalized plane-strain deformation superposed on the classical elastic solution. The governing equations for $\underset{\underset{u}{u}}{(1)}$ is thus derived by substituting Eq. 5-1 into Eq. 2-15. Since the classical solution satisfies Eq. 2-15 already, we obtain:

$(\lambda+2 \mu)\left(1-\ell_{1}^{2} \nabla^{2}\right) \nabla \nabla \cdot \underline{u}^{(1)}-\mu\left(1-\ell_{2}^{2} \nabla^{2}\right) \nabla \times \nabla \times \underline{u}^{(1)}=0$

The boundary conditions for ${\underset{\sim}{u}}^{(1)}$ is obtained by substituting Eq. 5-1 into Eq. 2-22. First, the stress field $\underset{\sim}{\tilde{I}}$ for $\underset{\sim}{u}$ of Eq. 5-1 is:

$$
\begin{aligned}
& \bar{\tau}_{r r}=\bar{\tau}_{r r}^{(1)} \\
& \bar{\tau}_{\theta \theta}=\bar{\tau}_{\theta \theta}^{(1)} \\
& \bar{\tau}_{z z}=\frac{E r}{\rho} \cos \theta+\bar{\tau}_{z z}^{(1)} \\
& \bar{\tau}_{r \theta}=\bar{\tau}_{r \theta}^{(1)} \\
& \bar{\tau}_{r z}=\bar{\tau}_{\theta z}=0
\end{aligned}
$$


where $\bar{I}^{(1)}$ denotes the stress tensor corresponding to the plane-strain deformation ${\underset{\sim}{u}}^{(1)}$. By the same notation, we have the couple-stress $\bar{\mu}$ :

$$
\begin{aligned}
& \bar{\mu}_{\theta z}=-\left[4 \nu \overline{\mathrm{d}}_{1}+4 \overline{\mathrm{d}}_{2}-\frac{1}{3}(1-4 \nu) \overline{\mathbf{f}}\right] \frac{\cos \theta}{\rho}+\bar{\mu}_{\theta z}^{(1)} \\
& \bar{\mu}_{z \theta}=-\left[4 \overline{\mathrm{d}}_{1}+4 v \overline{\mathrm{d}}_{2}+\frac{1}{3}(1-4 \nu) \overline{\mathrm{f}}\right] \frac{\cos \theta}{\rho}+\bar{\mu}_{z \theta}^{(1)} \\
& \bar{\mu}_{\mathrm{rz}}=-\left[4 \nu \overline{\mathrm{d}}_{1}+4 \overline{\mathrm{d}}_{2}-\frac{1}{3}(1-4 \nu) \overline{\mathrm{f}}\right] \frac{\sin \theta}{\rho}+\bar{\mu}_{\mathrm{rz}}^{(1)} \\
& \bar{\mu}_{z \mathrm{r}}=-\left[4 \overline{\mathrm{d}}_{1}+4 v \overline{\mathrm{d}}_{2}+\frac{1}{3}(1-4 \nu) \overline{\mathbf{f}}\right] \frac{\sin \theta}{\rho}+\bar{\mu}_{\mathrm{zr}}^{(1)} \\
& \bar{\mu}_{\mathrm{rr}}=\bar{\mu}_{\theta \theta}=\bar{\mu}_{z z}=\bar{\mu}_{r \theta}=\bar{\mu}_{\theta \mathrm{r}}=0
\end{aligned}
$$

and the double-stress $\bar{\mu}$ :

$$
\begin{aligned}
& \overline{\bar{\mu}}_{r r r}=\left[(1-4 v) \bar{a}_{1}-2 v \bar{a}_{2}+(1-v) \bar{f}\right] \frac{\cos \theta}{\rho}+\bar{\mu}_{r r r}^{(1)} \\
& \bar{\mu}_{r \theta \theta}=\left[(1-4 v) \bar{a}_{1}-2 v \bar{a}_{2}+(1-v) \bar{f}\right] \frac{\cos \theta}{3 \rho}+\bar{\mu}_{r \theta \theta}^{(1)} \\
& \overline{\bar{\mu}}_{r z z}=\left[(1-4 v) \bar{a}_{1}+2 \bar{a}_{2}+(1-v) \bar{f}\right] \frac{\cos \theta}{3 \rho}+\bar{\mu}_{r z z}^{(1)}
\end{aligned}
$$




$$
\begin{aligned}
& \overline{\bar{\mu}}_{\theta \mathrm{rr}}=-\left[(1-4 v) \overline{\mathbf{a}}_{1}-2 v \overline{\mathbf{a}}_{2}+(1-v) \overline{\mathbf{f}}\right] \frac{\sin \theta}{3 \rho}+\bar{\mu}_{\theta \mathrm{rr}}(1) \\
& \overline{\bar{\mu}}_{\theta \theta \theta}=-\left[(1-4 \nu) \overline{\mathrm{a}}_{1}-2 v \overline{\mathrm{a}}_{2}+(1-\mu) \overline{\mathbf{f}}\right] \frac{\sin \theta}{\rho}+\bar{\mu}_{\theta \theta \theta}(1) \\
& \overline{\bar{\mu}}_{\theta z z}=-\left[(1-4 \nu) \bar{a}_{1}+2 \bar{a}_{2}+(1-v) \overline{\mathbf{f}}\right] \frac{\sin \theta}{3 \rho}+\bar{\mu}_{\theta z z}(1) \\
& \overline{\bar{\mu}}_{r \theta z}=\bar{\mu}_{z r r}=\bar{\mu}_{z \theta \theta}=\bar{\mu}_{z z z}=0
\end{aligned}
$$

Substituting Eqs. 5-3, 5-4, and 5-5 into Eq. 2-22 and letting the left hand sides of Eq. 2-22 equal to zero, we then obtain the following boundary conditions for the free traction boundary conditions:

$$
\begin{aligned}
& 0=P_{r}^{(1)}+\left[(1-4 \nu) \bar{a}_{1}-2 v \bar{a}_{2}+(1-v) \bar{f}\right] \frac{\cos \theta}{a \rho} \\
& 0=P_{\theta}^{(1)}+\left[(1-4 \nu) \bar{a}_{1}-2 v \bar{a}_{2}+(1-v) \bar{f}\right] \frac{\sin \theta}{a \rho} \\
& 0=\bar{Q}_{z}^{(1)}-\left[2(1-4 v) \bar{a}_{1}-4 v \bar{a}_{2}+12 v \bar{d}_{1}+12 \bar{d}_{2}+(1+2 v) \bar{f}\right] \frac{\sin \theta}{3 p} \\
& 0=\bar{R}^{(1)}+\left[(1-4 \nu) \bar{a}_{1}-2 v \bar{a}_{2}+(1-\nu) \bar{f}\right] \frac{\cos \theta}{\rho} \\
& \bar{P}_{z}=\bar{Q}_{\theta}=0
\end{aligned}
$$


where $\overline{\mathrm{P}}_{\mathrm{r}}^{(1)}, \overline{\mathrm{P}}_{\theta}^{(1)}, \overline{\mathrm{Q}}_{\mathrm{z}}^{(1)}$, and $\overline{\mathrm{R}}^{(1)}$ denote functions of $\underset{\sim}{\mathrm{u}}(1)$ as indicated on the right hand sides of Eqs. $2-22_{1}, 2-22_{2}, 2-22_{5}$, and $2-22_{6}$.

Thus we have obtained the set of governing equations and boundary conditions for completely characterizing the superposing deformation ${\underset{\sim}{u}}^{(1)}$.

\subsection{Solution}

In this subsection, we discuss the solution for the boundary value problem.

In order to find the solution $\underset{\sim}{\underset{u}{(1)}}{ }^{(1)}$ we operate Eq. 5-2, by the divergence and the curl operators respectively to yield two fifth order partial differential equations:

$$
\begin{aligned}
& \left(1-\ell_{I}^{2} \nabla^{2}\right) \nabla^{2}\left[\frac{1}{r} \frac{\partial}{\partial r}\left(r u_{r}^{(1)}\right)+\frac{1}{r} u_{\theta, \theta}^{(1)}\right]=0 \\
& \left(1-\ell \frac{2}{2} \nabla^{2}\right) \nabla^{2}\left[\frac{1}{r} \frac{\partial}{\partial r}\left(r u_{\theta}^{(1)}\right)-\frac{1}{r} u_{\theta, \theta}^{(1)}\right]=0
\end{aligned}
$$

where $\nabla^{2}=\frac{\partial^{2}}{\partial r^{2}}+\frac{1}{r} \frac{\partial}{\partial r}+\frac{1}{r^{2}} \frac{\partial^{2}}{\partial \theta^{2}}$. Intuitively, Eqs. 5-7 1 and $5-7_{2}$ are the necessary conditions for $\underset{\sim}{u}(1)$ being solution of Eq. 5-2.

According to the boundary conditions in Eq. 5-6, the solution for Eq. 5-7 is a linear combination of $\sin \theta$ and $\cos \theta$. In a simply connected cylinder, this solution is given by: 


$$
\begin{aligned}
& u_{r}^{(1)}=\left\{A_{1} \ell_{1}^{3}\left[I_{0}\left(\frac{r}{\ell_{1}}\right)-\frac{\ell_{1}}{r} I_{1}\left(\frac{r}{\ell_{1}}\right)-\frac{1}{2}\right]-C_{2} \ell_{2}^{3}\left[\frac{\ell_{2}}{r} I_{1}\left(\frac{r}{\ell_{2}}\right)-\frac{1}{2}\right]+\right. \\
& \left.E_{1} r^{2}\right\} \cos \theta+\left\{A_{2} \ell_{2}^{3}\left[\frac{\ell_{2}}{r} I_{1}\left(\frac{r}{\ell_{2}}\right)-\frac{1}{2}\right]+C_{1} \ell_{1}^{3}\left[I_{0}\left(\frac{r}{\ell_{1}}\right)-\frac{\ell_{1}}{r} I_{1}\left(\frac{r}{\ell_{1}}\right)-\right.\right. \\
& \left.\left.\frac{1}{2}\right]+E_{2} r^{2}\right\} \sin \theta \text {, } \\
& u_{\theta}^{(1)}=\left\{-A_{1} \ell_{1}^{\beta}\left[\frac{\ell_{1}}{r} I_{1}\left(\frac{r}{\ell_{1}}\right)-\frac{1}{2}\right]+C_{2} \ell_{2}^{3}\left[I_{0}\left(\frac{r}{\ell_{2}}\right)-\frac{\ell_{2}}{r} I_{1}\left(\frac{r}{\ell_{2}}\right)-\frac{1}{2}\right]+\right. \\
& \left.E_{3} r^{2}\right\} \sin \theta+\left\{A_{2} \ell_{2}^{3}\left[I_{0}\left(\frac{r}{\ell_{2}}\right)-\frac{\ell_{2}}{r} I_{1}\left(\frac{r}{\ell_{2}}\right)-\frac{1}{2}\right]+\right. \\
& \left.C_{1} \ell_{1}^{3}\left[\frac{\ell_{1}}{r} I_{1}\left(\frac{r}{\ell_{1}}\right)-\frac{1}{2}\right]+E_{4} r^{2}\right\} \cos \theta \text {. }
\end{aligned}
$$

The plane deformation given by Eq. 5-8 satisfies the continuous differentibility in the cylinder.

The sufficient conditions for $\underset{\sim}{\mathrm{u}}(1)$ as given by Eq. 5-8 being a solution of Eq. 5-2 are obtained by substituting Eq. 5-8 into Eq. 5-2. This operation yields:

$(\mu-\lambda) E_{3}=(3 \lambda+5 \mu) E_{1}$,

$-(\mu-\lambda) E_{4}=(3 \lambda+5 \mu) E_{2}$.

With Eq. 5-9, we have only six constants of integration to be determined by the boundary conditions (Eq. 5-6). 
Using the boundary conditions (Eq. 5-6) at $r=a$, we obtain:

$$
\mathrm{A}_{2}=\mathrm{C}_{1}=\mathrm{E}_{2}=0
$$

and, after some simplifications, the algebraic equations for $A_{1}, C_{2}$, and $E_{1}$ are:

$$
\begin{aligned}
& \rho A_{1}\left\{\left(\lambda \ell_{1}^{2}-18 \bar{a}_{2} \frac{\ell^{2}}{a^{2}}\right) \frac{\ell_{1}}{a} I_{0}\left(\frac{a}{l_{1}}\right)+\left[(2 \mu-\lambda) \ell_{1}^{2}+4 \bar{a}_{2}+\right.\right. \\
& \left.\left.36 \overline{\mathrm{a}}_{2} \frac{\ell_{1}^{2}}{\mathrm{a}^{2}}\right] \frac{\ell_{1}^{2}}{\mathrm{a}^{2}} \mathrm{I}_{1}\left(\frac{\mathrm{a}}{\ell_{1}}\right)\right\}+\rho \mathrm{C}_{2}\left\{-\left(2 \mu \ell_{2}^{2}+18 \overline{\mathrm{a}}_{2} \frac{\ell_{2}^{2}}{\mathrm{a}^{2}}\right) \frac{\ell_{2}}{\mathrm{a}} \mathrm{I}_{0}\left(\frac{\mathrm{a}}{l_{2}}\right)+\right. \\
& \left.\left(\mu \ell \frac{2}{2}+6 \bar{a}_{2}+3 \bar{d}_{1}-\frac{\bar{f}}{2}+36 \bar{a}_{2} \frac{l_{2}^{2}}{a^{2}}\right) \frac{\ell_{2}^{2}}{a^{2}} I_{1}\left(\frac{a}{l_{2}}\right)\right\}+ \\
& 4 \rho E_{1} \text { a }\left\{\mu \frac{\lambda+\mu}{\mu-\lambda}+\left(2 \bar{a}_{1}+\bar{a}_{2}+\frac{\lambda+2 \mu}{\mu-\lambda} \bar{f}\right) \frac{1}{a^{2}}\right\}= \\
& -\frac{1}{a}\left[(1-4 \nu) a_{1}-2 v \bar{a}_{2}+(1-v) \bar{f}\right] \text {, }
\end{aligned}
$$

$$
\begin{aligned}
\rho A_{1}\left\{\lambda \frac{\ell_{1}^{3}}{a} I_{0}\left(\frac{a}{\ell_{1}}\right)-\left(2 \lambda \ell_{1}^{2}-\frac{3}{2} \bar{f}\right) \frac{\ell_{1}^{2}}{a^{2}} I_{1}\left(\frac{a}{\ell_{1}}\right)\right\}+\rho C_{2}\left\{\mu \frac{\ell_{1}^{3}}{a} I_{0}\left(\frac{a}{\ell_{2}}\right)-\right. \\
\left.\left(2 \mu \ell_{2}^{2}-3 \bar{d}_{1}+\frac{\bar{f}}{2}\right) \frac{l_{2}^{2}}{a^{2}} I_{1}\left(\frac{a}{\ell_{2}}\right)\right\}+4 \rho E_{1} a\left\{\mu \frac{\lambda+\mu}{\mu-\lambda}+\right. \\
\left.\left(\bar{f}+6 \bar{d}_{1} \frac{\lambda+2 \mu}{\mu-\lambda}\right) \frac{1}{a^{2}}\right\}=\frac{1}{a}\left[6\left(\nu \bar{d}_{1}+\bar{d}_{2}\right)-\frac{1-4 \nu}{2} \bar{f}\right],
\end{aligned}
$$




$$
\begin{gathered}
\rho A_{1}\left\{\left(2 \mu \ell_{1}^{2}+24 \bar{a}_{2} \frac{\ell_{1}^{2}}{a^{2}}\right) \frac{\ell_{1}}{a} I_{0}\left(\frac{a}{l_{1}}\right)-\left(4 \mu \ell_{1}^{2}+6 \bar{a}_{2}+48 \bar{a}_{2} \frac{\ell_{1}^{2}}{a^{2}}\right) \frac{\ell_{1}^{2}}{a^{2}} I_{1}\left(\frac{a}{\ell_{1}}\right)\right\}+ \\
\quad \rho C_{2}\left\{\left(2 \mu \ell_{2}^{2}+24 \bar{a}_{2} \frac{l_{2}^{2}}{a^{2}}\right) \frac{\ell_{2}}{a} I_{0}\left(\frac{a}{l_{2}}\right)-\left(4 \mu l_{2}^{2}+6 \bar{a}_{2}+\right.\right. \\
\left.\left.48 \bar{a}_{2} \frac{l_{2}^{2}}{a^{2}}\right) \frac{l_{2}^{2}}{a^{2}} I_{1}\left(\frac{a}{l_{2}}\right)\right\}-4 \mu \rho E_{1} a \frac{\lambda+\mu}{\mu-\lambda}=0 .
\end{gathered}
$$

In Eq. 5-11 we have assumed that $\mu \neq \lambda$. If $\mu=\lambda$, we then have $3 \lambda+5 \mu \neq 0$ and $E_{1}=0$ from Eq. 5-9 . Therefore, the indeterminate quotient $E_{1} /(\mu-\lambda)$ in Eq. 5-11 shall be replaced by $E_{3} /(3 \lambda+5 \mu)$.

The analytical expressions for $A_{1}, C_{2}$, and $E_{1}$ are cumbersome due to the complexity of Eq. 5-11. The solution, nevertheless, is straightforward and theoretically easy to obtain from Eq. 5-11.

Finally, we note that Eqs. 5-6 and 5-62 yield the identical equations under conditions in Eq. 5-9 and hence only three equations for the determination of $A_{1}, C_{2}$, and $E_{1}$ result and no overdetermination for the integration constants. We also note that $A_{1}, C_{2}$, and $E_{1}$ are linear functions of $\overline{\mathrm{d}}_{2}$ which appears only on the right hand side of $\mathrm{Eq} .5-11_{2}$ and that these constants are inversely proportional to the bending curvature $1 / p$.

\subsection{Stress and Bending Moment}

For the stress tensor $\bar{\tau}$, the couple-stress tensor $\bar{\mu}$ and the doublestress tensor $\underset{\sim}{\bar{\mu}}$, we will need $\underset{\tau}{\tau}(1), \underset{\mu}{\sim}(1)$, and $\underset{\sim}{\bar{\mu}}(1)$ as shown by Eqs. 5-3, $5-4$, and 5-5. By a routine substitution, noting that $u_{r}^{(1)}$ and $u_{\theta}^{(1)}$ are functions of $r$ and $\theta$ solely and $u_{z}^{(1)}=0$, we obtain $\bar{\tau}^{(1)},{\underset{\mu}{\mu}}^{(1)}$, and $\overline{\bar{\mu}}(1)$ from Eqs. $2-4$ and $2-6$. The entries of $\underset{\sim}{\tau}(1)$ are 


$$
\begin{aligned}
& \bar{\tau}_{\mathrm{rr}}^{(1)}=\left\{\mathrm{A}_{1} \ell_{\mathrm{I}}^{2}\left[-2 \mu \frac{\ell_{1}}{\mathrm{r}} \mathrm{I}_{0}\left(\frac{\mathrm{r}}{\ell_{1}}\right)+\left(2 \mu+\lambda+4 \mu \frac{\ell_{1}^{2}}{\mathrm{r}^{2}}\right) \mathrm{I}_{1}\left(\frac{\mathrm{r}}{\ell_{1}}\right)\right]+\right. \\
& \mathrm{C}_{2} \ell \frac{2}{2}\left[-2 \mu \frac{\ell_{2}}{\mathrm{r}} \mathrm{I}_{0}\left(\frac{\mathrm{r}}{\ell_{2}}\right)+4 \mu \frac{\ell_{2}^{2}}{\mathrm{r}^{2}} \mathrm{I}_{1}\left(\frac{\mathrm{r}}{\ell_{2}}\right)\right]+ \\
& \left.E_{1} r\left[4 \mu \frac{\mu+\lambda}{\mu-\lambda}\right]\right\} \cos \theta \text {. } \\
& \bar{\tau}_{\theta \theta}^{(1)}=\left\{A_{1} \ell_{1}^{2}\left[2 \mu \frac{\ell_{1}}{r} I_{0}\left(\frac{r}{\ell_{1}}\right)+\left(\lambda-4 \mu \frac{\ell_{1}^{2}}{r^{2}}\right) I_{1}\left(\frac{r}{\ell_{1}}\right)\right]+\right. \\
& \mathrm{C}_{2} \ell_{2}^{2}\left[2 \mu \frac{\ell_{2}}{\mathrm{r}} \mathrm{I}_{0}\left(\frac{\mathrm{r}}{\ell_{2}}\right)-4 \mu \frac{\ell_{2}^{2}}{\mathrm{r}^{2}} \mathrm{I}_{1}\left(\frac{\mathrm{r}}{\ell_{1}}\right)\right]+ \\
& \left.E_{1} r\left[12 \mu \frac{\mu+\lambda}{\mu-\lambda}\right]\right\} \cos \theta \text {, } \\
& \bar{\tau}_{z z}^{(1)}=\left\{A_{1} \ell_{1}^{2} \lambda I_{1}\left(\frac{r}{\ell_{1}}\right)+\frac{8 \lambda \mu}{\mu-\lambda} E_{1} r\right\} \cos \theta, \\
& \underset{\mathrm{r} \theta}{\bar{\tau}(1)}=\left\{\mathrm{A}_{1} \ell_{1}^{2}\left[-2 \mu \frac{\ell_{1}}{\mathrm{r}} \mathrm{I}_{0}\left(\frac{\mathrm{r}}{\ell_{1}}\right)+4 \mu \frac{\ell_{1}^{2}}{\mathrm{r}^{2}} \mathrm{I}_{1}\left(\frac{\mathrm{r}}{\ell_{1}}\right)\right]+\right. \\
& \mathrm{C}_{2} \ell \frac{2}{2}\left[-2 \mu \frac{\ell_{2}}{\mathrm{r}} \mathrm{I}_{0}\left(\frac{\mathrm{r}}{\ell_{2}}\right)+\mu\left(1+4 \frac{\ell_{2}^{2}}{\mathrm{r}^{2}}\right) \mathrm{I}_{l}\left(\frac{\mathrm{r}}{\ell_{2}}\right)\right]+ \\
& \left.4 \mu \frac{\mu+\lambda}{\mu-\lambda} E_{1} r\right\} \sin \theta \text {, } \\
& \bar{\tau}_{r z}^{(1)}=\frac{\tau_{\theta z}^{(1)}}{\tau^{2}}=0
\end{aligned}
$$


The couple-stress $\bar{\sim}^{(1)}$ has the following entries:

$\bar{\mu}_{r r}^{(1)}=\bar{\mu}_{\theta \theta}^{(1)}=\bar{\mu}_{z z}^{(1)}=\bar{\mu}_{r \theta}^{(1)}=\bar{\mu}_{\theta \mathbf{r}}^{(1)}=0$,

$\bar{\mu}_{\theta \mathrm{Z}}^{(1)}=\left\{\mathrm{A}_{1} \ell_{1} \overline{\mathrm{f}}\left[\mathrm{I}_{0}\left(\frac{\mathrm{r}}{\ell_{1}}\right)-\frac{\ell_{1}}{\mathrm{r}} \mathrm{I}_{1}\left(\frac{\mathrm{r}}{\ell_{1}}\right)\right]+\mathrm{C}_{2} \ell_{2}\left(2 \overline{\mathrm{d}}_{1}-\frac{\overline{\mathrm{f}}}{3}\right) \frac{\ell_{2}}{\mathrm{r}} \mathrm{I}_{1}\left(\frac{\mathrm{r}}{\ell_{2}}\right)+\right.$ $\left.E_{1}\left(16 \frac{2 \mu+\lambda}{\mu-\lambda} \bar{d}_{1}+\frac{8}{3} \bar{f}\right)\right\} \cos \theta$,

$\bar{\mu}_{z \theta}^{(1)}=\left\{-A_{1} \ell_{1} \bar{f}\left[I_{0}\left(\frac{r}{\ell_{1}}\right)-\frac{\ell_{1}}{r} I_{1}\left(\frac{r}{\ell_{1}}\right)\right]+C_{2} \ell_{2}\left(2 \bar{d}_{2}+\frac{\bar{f}}{3}\right) \frac{\ell_{2}}{r} I_{1}\left(\frac{r}{\ell_{2}}\right)+\right.$ $\left.E_{1}\left(16 \frac{2 \mu+\lambda}{\mu-\lambda} \bar{d}_{2}-\frac{8}{3} \bar{f}\right)\right\} \cos \theta$,

$\bar{\mu}_{\mathrm{rz}}^{(1)}=\left\{\mathrm{A}_{1} \ell_{1} \overline{\mathrm{f}} \frac{\ell_{1}}{\mathrm{r}} \mathrm{I}_{1}\left(\frac{\mathrm{r}}{\ell_{1}}\right)+\mathrm{C}_{2} \ell_{2}\left(2 \overline{\mathrm{d}}_{1}-\frac{\overline{\mathrm{f}}}{3}\right)\left[\mathrm{I}_{0}\left(\frac{\mathrm{r}}{\ell_{2}}\right)-\frac{\ell_{2}}{\mathrm{r}} \mathrm{I}_{1}\left(\frac{\mathrm{r}}{\ell_{2}}\right)\right]+\right.$ $\left.\mathrm{E}_{1}\left(16 \frac{2 \mu+\lambda}{\mu-\lambda} \overline{\mathrm{d}}_{1}+\frac{8}{3} \overline{\mathrm{f}}\right)\right\} \sin \theta$,

$\bar{\mu}_{z \mathrm{r}}^{(1)}=\left\{-\mathrm{A}_{1} \ell_{1} \overline{\mathrm{f}} \frac{\ell_{1}}{\mathrm{r}} \mathrm{I}_{1}\left(\frac{\mathrm{r}}{\ell_{1}}\right)+\mathrm{C}_{2} \ell_{2}\left(2 \overline{\mathrm{d}}_{2}+\frac{\overline{\mathrm{f}}}{3}\right)\left[\mathrm{I}_{0}\left(\frac{\mathrm{r}}{\ell_{2}}\right)-\frac{\ell_{2}}{\mathrm{r}} \mathrm{I}_{1}\left(\frac{\mathrm{r}}{\ell_{2}}\right)\right]+\right.$ $\left.E_{1}\left(16 \frac{2 \mu+\lambda}{\mu-\lambda} \bar{d}_{2}-\frac{8}{3} \bar{f}\right)\right\} \sin \theta$ 
Finally, the entries of the double-stress $\underset{\sim}{\bar{\mu}}(1)$ are

$$
\begin{aligned}
& \overline{\bar{\mu}}_{\mathrm{r} \theta \mathrm{z}}^{(1)}=\underset{\bar{\mu}_{\mathrm{zrr}}}{(1)}=\overline{\bar{\mu}}_{\mathrm{z} \theta \theta}^{(1)}=\overline{\bar{\mu}}_{\mathrm{zzz}}^{(1)}=0 \text {, }
\end{aligned}
$$

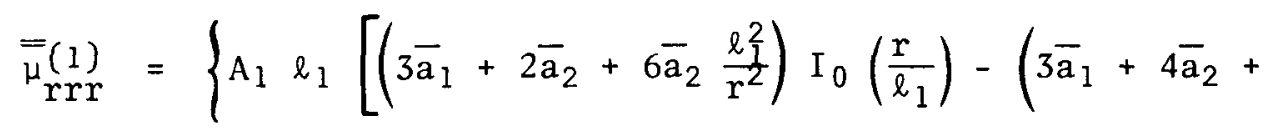

$$
\begin{aligned}
& \left.\left.12 \overline{\mathrm{a}}_{2} \frac{\ell_{1}^{2}}{\mathrm{r}^{2}}\right) \frac{\ell_{1}}{\mathrm{r}} \mathrm{I}_{1}\left(\frac{\mathrm{r}}{\ell_{1}}\right)\right]+\mathrm{C}_{2} \ell_{2}\left[6 \overline{\mathrm{a}}_{2} \frac{\ell_{2}^{2}}{\mathrm{r}^{2}} \mathrm{I}_{0}\left(\frac{\mathrm{r}}{\ell_{2}}\right)-\left(\overline{\mathrm{a}}_{1}+2 \overline{\mathrm{a}}_{2}-\right.\right. \\
& \left.\left.\left.\overline{\frac{\mathrm{f}}{2}}+12 \overline{\mathrm{a}}_{2} \frac{\ell_{2}^{2}}{\mathrm{r}^{2}}\right) \frac{\ell_{2}}{\mathrm{r}} \mathrm{I}_{1}\left(\frac{\mathrm{r}}{\ell_{2}}\right)\right]+4 \mathrm{E}_{3}\left(2 \overline{\mathrm{a}}_{1}+\overline{\mathrm{a}}_{2}+\frac{2 \mu+\lambda}{\mu-\lambda} \overline{\mathrm{f}}\right)\right\} \cos \theta,
\end{aligned}
$$$$
\underset{\mu_{r \theta \theta}}{\bar{a}(1)}=\left\{A_{1} \ell_{1}\left[\left(\bar{a}_{1}-6 \bar{a}_{2} \frac{\ell_{1}^{2}}{r^{2}}\right) I_{0}\left(\frac{r}{l_{1}}\right)-\left(\bar{a}_{1}-2 \bar{a}_{2}-12 \bar{a}_{2} \frac{\ell_{1}^{2}}{r^{2}}\right) \frac{\ell_{1}}{r} I_{1}\left(\frac{r}{l_{1}}\right)\right]-\right.
$$$$
\mathrm{C}_{2} \ell_{2}\left[6 \overline{\mathrm{a}}_{2} \frac{\ell_{2}^{2}}{\mathrm{r}^{2}} \mathrm{I}_{0}\left(\frac{\mathrm{r}}{l_{2}}\right)+\left(\frac{\overline{\mathrm{a}}_{1}}{3}-\frac{4}{3} \overline{\mathrm{a}}_{2}-\frac{\overline{\mathrm{f}}}{6}-12 \overline{\mathrm{a}}_{2} \frac{\ell_{2}^{2}}{\mathrm{r}^{2}}\right) \frac{\ell_{2}}{\mathrm{r}} \mathrm{I}_{1}\left(\frac{\mathrm{r}}{l_{2}}\right)\right]+
$$$$
\left.\frac{4}{3} E_{1}\left(2 \bar{a}_{1}+\bar{a}_{2}+\frac{2 \mu+\lambda}{\mu-\lambda} \bar{f}\right)\right\} \cos \theta,
$$$$
\underset{\mathrm{\mu} Z \mathrm{Z}}{\overline{\mathrm{u}}(1)}=\left\{\mathrm{A}_{1} \ell_{1} \overline{\mathrm{a}}_{1}\left[\mathrm{I}_{0}\left(\frac{\mathrm{r}}{\ell_{1}}\right)-\frac{\ell_{1}}{\mathrm{r}} \mathrm{I}_{1}\left(\frac{\mathrm{r}}{\ell_{1}}\right)\right]-\frac{1}{6} \mathrm{C}_{2} \ell_{2}\left(2 \overline{\mathbf{a}}_{1}-\overline{\mathrm{f}}\right) \frac{\ell_{2}}{\mathrm{r}} \mathrm{I}_{1}\left(\frac{\mathrm{r}}{\ell_{2}}\right)+\right.
$$$$
\left.\frac{4}{3} E_{1}\left(2 \vec{a}_{1}+\frac{2 \mu+\lambda}{\mu-\lambda} \vec{f}\right)\right\} \cos \theta
$$ 


$$
\begin{aligned}
& \overline{\bar{\mu}}(1)=\left\{A_{1} \quad \ell_{1}\left[6 \bar{a}_{2} \frac{\ell_{1}^{2}}{r^{2}} I_{0}\left(\frac{r}{\ell_{1}}\right)-\left(\bar{a}_{1}+2 \bar{a}_{2}+12 \bar{a}_{2} \frac{\ell_{1}^{2}}{r^{2}}\right) I_{1}\left(\frac{r}{\ell_{1}}\right)\right]+\right. \\
& C_{2} \ell_{2}\left[\left(\frac{\bar{a}_{1}}{3}+\frac{2}{3} \bar{a}_{2}-\frac{\bar{f}}{6}+6 \bar{a}_{2} \frac{l_{2}^{2}}{\mathrm{r}^{2}}\right) I_{0}\left(\frac{\mathrm{r}}{l_{2}}\right)-\left(\frac{\bar{a}_{1}}{3}+\frac{8}{3} \bar{a}_{2}-\right.\right. \\
& \left.\left.\left.\frac{\overline{\mathrm{f}}}{6}+12 \overline{\mathrm{a}}_{2} \frac{\ell_{3}^{2}}{\mathrm{r}^{2}}\right) \frac{\ell_{2}}{\mathrm{r}} \mathrm{I}_{1}\left(\frac{\mathrm{r}}{\ell_{2}}\right)\right]-\frac{4}{3} \mathrm{E}_{1}\left(2 \overline{\mathrm{a}}_{1}+\overline{\mathrm{a}}_{2}+\frac{2 \mu+\lambda}{\mu-\lambda} \overline{\mathrm{f}}\right)\right\} \sin \theta, \\
& \overline{\bar{\mu}_{\theta \theta \theta}^{(1)}}=\left\{A_{1} \ell_{1}\left[-6 \bar{a}_{2} \frac{\ell_{1}^{2}}{r^{2}} I_{0}\left(\frac{r}{\ell_{1}}\right)-3\left(\bar{a}_{1}-4 \bar{a}_{2} \frac{\ell_{1}^{2}}{r^{2}}\right) \frac{\ell_{1}}{r} I_{1}\left(\frac{r}{\ell_{1}}\right)\right]+\right. \\
& \mathrm{C}_{2} \ell_{2}\left[\left(\overline{\mathrm{a}}_{1}-\frac{\overline{\mathrm{f}}}{2}-6 \overline{\mathrm{a}}_{2} \frac{\mathrm{l}_{2}^{2}}{\mathrm{r}^{2}}\right) I_{0}\left(\frac{\mathrm{r}}{\mathrm{l}_{2}}\right)+\left(-\overline{\mathrm{a}}_{1}+2 \overline{\mathrm{a}}_{2}+\frac{\overline{\mathrm{f}}}{2}+\right.\right. \\
& \left.\left.\left.12 \bar{a}_{2} \frac{\ell_{2}^{2}}{r^{2}}\right) \frac{\ell_{2}}{r} I_{1}\left(\frac{r}{l_{2}}\right)\right]-4 E_{1}\left(2 \bar{a}_{1}+\bar{a}_{2}+\frac{2 \mu+\lambda}{\mu-\lambda} \bar{f}\right)\right\} \sin \theta \text {, } \\
& \overline{\overline{\mu_{\theta z z}}}(1)=\left\{-A_{1} \ell_{1} \bar{a}_{1} \frac{\ell_{1}}{r} I_{1}\left(\frac{r}{\ell_{1}}\right)+C_{2} \ell_{2}\left(\frac{\overline{a_{1}}}{3}-\frac{\bar{f}}{6}\right)\left[I_{0}\left(\frac{r}{\ell_{2}}\right)-\frac{\ell_{2}}{r} I_{1}\left(\frac{r}{\ell_{2}}\right)\right]-\right. \\
& \left.\frac{4}{3} E_{1}\left(2 \bar{a}_{1}+\frac{2 \mu+\lambda}{\mu-\lambda}\right) \bar{f}\right\} \sin \theta .
\end{aligned}
$$

The couple-stress tensor $\underset{\sim}{\sim}$ given by Eq. 5-4 has that $\bar{\mu}_{\mathrm{rr}}=0$ on the cylinder surface. That is, the normal component of $\underset{\sim}{\sim}$ on the surface vanishes and agrees with the free traction boundary condition. Consequently, for the couple-stress field of the circular cylinder, we have

$\underline{\mu}=\underline{\mu}$ 
The actual stress tensor $\tau$ is obtained from Eq. $2-18$ with $\underset{\sim}{\tau}, \bar{\mu}$, and $\underset{\sim}{\sim}$ given by Eqs. 5-3, 5-4, and 5-5 with $\underset{\sim}{\tau}(1), \underset{\sim}{\mu}(1)$, and $\underset{\sim}{\mu}(1)$ given by Eqs. 5-12, 5-13, and 5-14. The entries of $\tau$ are

$$
\begin{aligned}
& \tau_{r r}=\left\{A_{1}\left(\lambda \ell_{1}^{2}-\bar{a}_{1}\right)\left[\frac{\ell_{1}}{r} I_{0}\left(\frac{r}{\ell_{1}}\right)-2 \frac{\ell_{1}^{2}}{r^{2}} I_{1}\left(\frac{r}{\ell_{1}}\right)\right]-\frac{1}{3} C_{2}\left(6 \bar{d}_{1}-\right.\right. \\
& \left.\bar{f})\left[\frac{l_{2}}{r} I_{0}\left(\frac{r}{l_{2}}\right)-2 \frac{l_{3}^{2}}{r^{2}} I_{1}\left(\frac{r}{l_{2}}\right)\right]\right\} \cos \theta \text {, } \\
& \tau_{\theta \theta}=\left\{A_{1}\left(\lambda \ell_{1}^{2}-\bar{a}_{1}\right)\left[\frac{\ell_{1}}{r} I_{0}\left(\frac{r}{\ell_{1}}\right)+\left(1+2 \frac{\ell_{1}^{2}}{r^{2}}\right) \frac{\ell_{1}^{2}}{r^{2}} I_{1}\left(\frac{r}{\ell_{1}}\right)\right]+C_{2}\left(6 \bar{d}_{1}-\right.\right. \\
& \left.\bar{f})\left[\frac{\ell_{2}}{3 r} I_{0}\left(\frac{r}{\ell_{2}}\right)-2 \frac{\ell_{2}^{2}}{r^{2}} I_{1}\left(\frac{r}{\ell_{2}}\right)\right]+12 \mu \frac{\mu+\lambda}{\mu-\lambda} E_{3} r\right\} \cos \theta \text {, } \\
& \tau_{z z}=\left\{\frac{E r}{\rho}+A_{1}\left(\lambda \ell_{1}^{2}-\bar{a}_{1}\right) I_{I}\left(\frac{r}{\ell_{1}}\right)+\frac{8 \lambda \mu}{\mu-\lambda}\right\} \cos \theta \\
& \tau_{\mathrm{r} \theta}=\left\{\mathrm{A}_{1}\left(\lambda \ell_{1}^{2}-\overline{\mathrm{a}}_{1}\right)\left[\frac{\ell_{1}}{\mathrm{r}} \mathrm{I}_{0}\left(\frac{\mathrm{r}}{\ell_{1}}\right)-2 \frac{\ell_{1}^{2}}{\mathrm{r}^{2}} \mathrm{I}_{1}\left(\frac{\mathrm{r}}{\ell_{1}}\right)\right]-\mathrm{C}_{2}\left(\overline{\mathrm{d}}_{1}-\right.\right. \\
& \left.\frac{\bar{f}}{6}\right)\left[2 \frac{\ell_{2}}{r} I_{0}\left(\frac{r}{\ell_{2}}\right)+\left(1-\frac{\ell_{2}^{2}}{r^{2}}-4 \frac{\ell_{2}^{4}}{r^{4}}\right) I_{1}\left(\frac{r}{\ell_{2}}\right)\right]+ \\
& \left.4 \mu \frac{\mu+\lambda}{\mu-\lambda} E_{1} r\right\} \sin \theta \text {, } \\
& \tau_{\theta r}=\left\{A_{1}\left(\lambda \ell_{1}^{2}-\bar{a}_{1}\right)\left[\frac{\ell_{1}}{r} I_{0}\left(\frac{r}{\ell_{1}}\right)-2 \frac{\ell_{1}^{2}}{r^{2}} I_{1}\left(\frac{r}{\ell_{1}}\right)\right]-C_{2}\left(\bar{d}_{1}-\right.\right. \\
& \left.\frac{\overline{\mathrm{f}}}{6}\right)\left[2 \frac{\ell_{2}}{\mathrm{r}} I_{0}\left(\frac{\mathrm{r}}{\ell_{2}}\right)-\left(1+\frac{\ell_{2}^{2}}{\mathrm{r}^{2}}+4 \frac{\ell_{2}^{4}}{\mathrm{r}^{4}}\right) I_{1}\left(\frac{\mathrm{r}}{\ell_{2}}\right)\right]+ \\
& \left.4 \mu \frac{\mu+\lambda}{\mu-\lambda} E_{1} r\right\} \sin \theta \text {, }
\end{aligned}
$$




$$
\tau_{\theta z}=\tau_{z \theta}=\tau_{r z}=\tau_{z r}=0
$$

We note the non-symmetry of the stress tensor $\tau$ as shown in Eq. 5-16 due to the presence of the couple-stresses.

The bending moment on the cylinder with the deformation field of Eq. 5-1 in the strain-gradient theory is the sum of the moment supported by the stress field of Eq. 5-16 and the couple-stress field of Eq. 5-15. There is no direct contribution of the double-stress in the bending moment. Let $\mathrm{A}$ be any cross section of the cylinder, the bending moment is then

$$
\stackrel{M}{\sim}=\int_{\mathrm{A}}[\underline{\mathrm{r}} \times(\underline{\sim} \cdot \underline{\tau})+\underline{\mathrm{n}} \cdot \underset{\sim}{\sim}] \mathrm{ds},
$$

where $\underset{\sim}{\sim}$ is the unit normal vector to the area element $d s$ which has a position vector $\underset{\sim}{r}$. If $\mathrm{A}$ is chosen to be an orthonormal cross section, then $\underset{\sim}{\sim}=\stackrel{e}{ }^{<z>}$, and hence Eqs. 5-4 and 5-16 yield that

$\underset{\sim}{\sim}=\int_{0}^{2 \pi} \int_{0}^{\mathrm{a}}\left(\tau_{z z} r \cos \theta+\mu_{z \mathbf{r}} \sin \theta+\mu_{z \theta} \cos \theta\right) r d \theta d r,(5-18)$

where $j$ is the unit vector in the $y$-axis direction. Substitute Eqs. 5-16, 5-4, and 5-13 into Eq. 5-18 and integrate as indicated, we obtain

$$
M_{x}=0,
$$




$$
\begin{gathered}
M_{y}=-\frac{E \pi a^{4}}{4 \rho}-\frac{\pi a^{2}}{\rho}\left[4 \bar{d}_{1}+4 \nu \bar{d}_{2}+\frac{1}{3}(1-4 \nu) \bar{f}\right]-\pi A_{1} \ell_{1}^{3}\left[\left(\frac{v E \ell_{1}^{2}}{(1+\nu)(1-2 \nu)}-\right.\right. \\
\left.\left.\bar{a}_{1}\right)\left\{\frac{a^{2}}{\ell_{1}^{2}} I_{0}\left(\frac{a}{\ell_{1}}\right)-2 \frac{a}{\ell_{1}} I_{1}\left(\frac{a}{\ell_{1}}\right)\right\}+\bar{f} \frac{a}{\ell_{1}} I_{1}\left(\frac{a}{\ell_{1}}\right)\right]+\pi C_{2} \ell_{2}^{3}\left(2 \bar{d}_{2}+\right. \\
\left.\frac{1}{3} \bar{f}\right) \frac{a}{\ell_{2}} I_{1}\left(\frac{a}{\ell_{2}}\right)+\pi a^{2} E_{1}\left(\frac{32(1-\nu)}{1-4 \nu} \bar{d}_{2}+\frac{8}{3} \bar{f}+\right. \\
\left.\frac{2 \nu E a^{2}}{(1+\nu)(1-4 \nu)}\right),
\end{gathered}
$$

where $M_{x}$ and $M_{y}$ are the bending moment components about $x$ - and $y$-axes, respectively, on the cross section.

We remark here that the stress $\tau$ is a linear function of $\bar{d}_{2}$ and the moment $\underset{\sim}{\mathcal{M}}$ is a quadratic function of $\overline{\mathrm{d}}_{2}$. Furthermore, both $\tau$ and $\underset{\sim}{\mathrm{M}}$ are proportional to the bending curvature $1 / \rho$. These facts follow from the properties of $A_{1}, C_{2}$, and $E_{1}$.

Finally, the solution and the stress fields presented in this section reduces to the pure-bending solution in the couple-stress theory (Ref. 1) if we let $\bar{a}_{1}=\bar{a}_{2}=\bar{f}=0$. 
REFERENCES

1. Kao, B. G., F. K. Tzung, and F. H. Ho, "Influences of the Couple-Stresses on the Pure-Bending of a Circular Cylinder," General Atomic report GA-A15141, July 23, 1979.

2. Ely, R. E., "Strength of Magnesium Silicate and Graphite under Biaxial Stresses," Ceramic Bulletin, 47, pp. 489 - 492, 1968.

3. Mindlin, R. D., "Micro-Structure in Linear Elasticity," Archive for Rational Mechanics and Analysis, 16, pp. 51 - 78, 1964.

4. Mindlin, R. D., and N. N. Eshel, "On First Strain-Gradient Theories in Linear Elasticity," International Journal of Solids and Structures, 4, pp. 109 - 124, 1968.

5. Mindlin, R. D., and H. F. Tiersten, "Effects of Couple-Stresses in Linear Elasticity," Archive for Rational Mechanics and Analysis, 11, pp. 415 - 448, 1962 .

6. Timoshenko, S. P., and J. N. Goodier, "Theory of Elasticity," 3rd ed., McGraw-Hi11, Inc., New York, 1970. 